\title{
Ethnicity and coercion among involuntarily detained psychiatric in-patients
}

Olive Bennewith, Tim Amos, Glyn Lewis, Christina Katsakou, Til Wykes, Richard Morriss and Stefan Priebe

\section{Summary}

We assessed whether adult Black and minority ethnic (BME) patients detained for involuntary psychiatric treatment experienced more coercion than similar White patients. We found no evidence of this from patient interviews or from hospital records. The area (mental health trust) where people were treated was strongly associated with both the experience of coercion and the recording of a coercive measure in their records. Regarding charges of institutional racism in psychiatry, this study highlights the importance of investigating the role of area characteristics when assessing the relationship between ethnicity and patient management.

\section{Declaration of interest}

None.
Accusations of institutional racism in psychiatry have been made for several years in the USA and UK. ${ }^{1-3}$ One area of investigation has been the use of in-patient coercion: three studies have reported higher levels of coercion among Black and ethnic minority (BME) psychiatric in-patients compared with White patients. ${ }^{4-6}$ However, in the one study that carried out more complex analyses, ethnic differences in coercion were explained by adjustment for factors that differed according to ethnic background. ${ }^{4}$ In our study of detained in-patients we obtained information from both self-report and hospital records, collected at different times during the hospital stay. Our hypothesis was that detained BME patients would experience more coercion than White patients.

\section{Method}

Twenty-two hospitals managed by eight mental health trusts, located in London and in the south-east, north-west and southwest of England, participated in a study of involuntary hospital admissions. ${ }^{7}$ Patients aged 18-65 years who had been admitted under Sections 2, 3 or 4 of the Mental Health Act 1983, or who became involuntary patients within a week of admission, were recruited between July 2003 and July 2005. Recruitment took place during the 7-day period following admission and interviews were carried out at baseline and 4 weeks after admission. Following approval by the Patient Information Advisory Group, demographic data were obtained for all eligible patients. Participants were asked to indicate their ethnicity using the 16 categories from the 2001 census. Owing to the small numbers in some categories, for analysis data on ethnicity were collapsed into four categories: White, Black, Asian and mixed ethnicity.

At baseline and 4 weeks after admission those consenting to be interviewed indicated on the Coercion Ladder, a ten-point visual analogue scale based on Cantril's Ladder, ${ }^{8,9}$ the level of coercion they had experienced (see online supplement). Information on forced medication, use of restraint and seclusion was obtained from participant interviews (online supplement) and from interviewees' records during the first 4 weeks of hospital stay.

Logistic regression models were used to estimate odds ratios for our outcomes and for adjustments. Three outcome variables were used: a patient score of 10 on the Coercion Ladder at baseline or at 4 weeks; a patient report of any coercive measure; and a record of a coercive measure in the notes. Statistical analyses were carried out using Stata version 9.0 for Windows.

\section{Results}

About half $(n=778)$ of the 1570 eligible patients consented to be interviewed. Recruitment was similar across ethnic groups (online Table DS1). Information on ethnicity was recorded for 773 of the 778 patients interviewed. Of these, $73.0 \%(n=564)$ were White, $17.9 \%(n=138)$ Black, $6.1 \%(n=47)$ Asian and $3.1 \%(n=24)$ of mixed ethnicity. Three-quarters of the participants of Black or Asian ethnicity were recruited from two hospitals managed by one mental health trust in inner London. Nearly three-quarters of patients $(70.5 \%, n=545)$ interviewed at baseline agreed to a second interview 4 weeks after admission. Retention rates were similar across ethnic groups (Table DS1). Both eligible patients and interviewees in the BME groups were younger, more likely to be male and to have a diagnosis of schizophrenia.

Many patients (249 of $716 ; 34.8 \%, 95 \%$ CI 31-38) reported that the maximum level of coercion had been used during their admission. Such reports were more frequent among Black patients (adjusted analyses, Table 1; unadjusted, Table DS2). However, this association was largely explained in terms of the mental health trust: trusts with higher than average coercion had more patients from ethnic minorities, and in the adjusted model we found an association between mental health trust and perception of coercion $\left(\chi^{2}=32.19\right.$, d.f. $\left.=7, P<0.01\right)$ but not between ethnicity and coercion. At 4 weeks, $15.6 \%$ (95\% CI 13-19) of participants reported maximum coercion. There was no evidence of association with ethnicity before or after adjustment (Tables 1 \& DS2). About half (52.1\%, 95\% CI 48-56) of the interviewees reported restraint, enforced medication or seclusion. There was no evidence for an association between ethnicity and use of these coercive measures. There was a significant association between mental health trust and use of a coercive measure after adjustment $\left(\chi^{2}=20.42\right.$, d.f. $\left.=7, P<0.01\right)$. Only a quarter (95\% CI $\left.22-28\right)$ of patients had a hospital record of a coercive measure. There was a difference in the use of such measures across ethnic groups, but not after adjustment. The two mental health trusts with the highest proportion of patients from ethnic minorities had more frequent use of coercive measures $\left(\chi^{2}=20.17\right.$, d.f. $=7$, $P<0.01)$. 
Table 1 Proportion and odds ratios of coercion, categorised by ethnicity

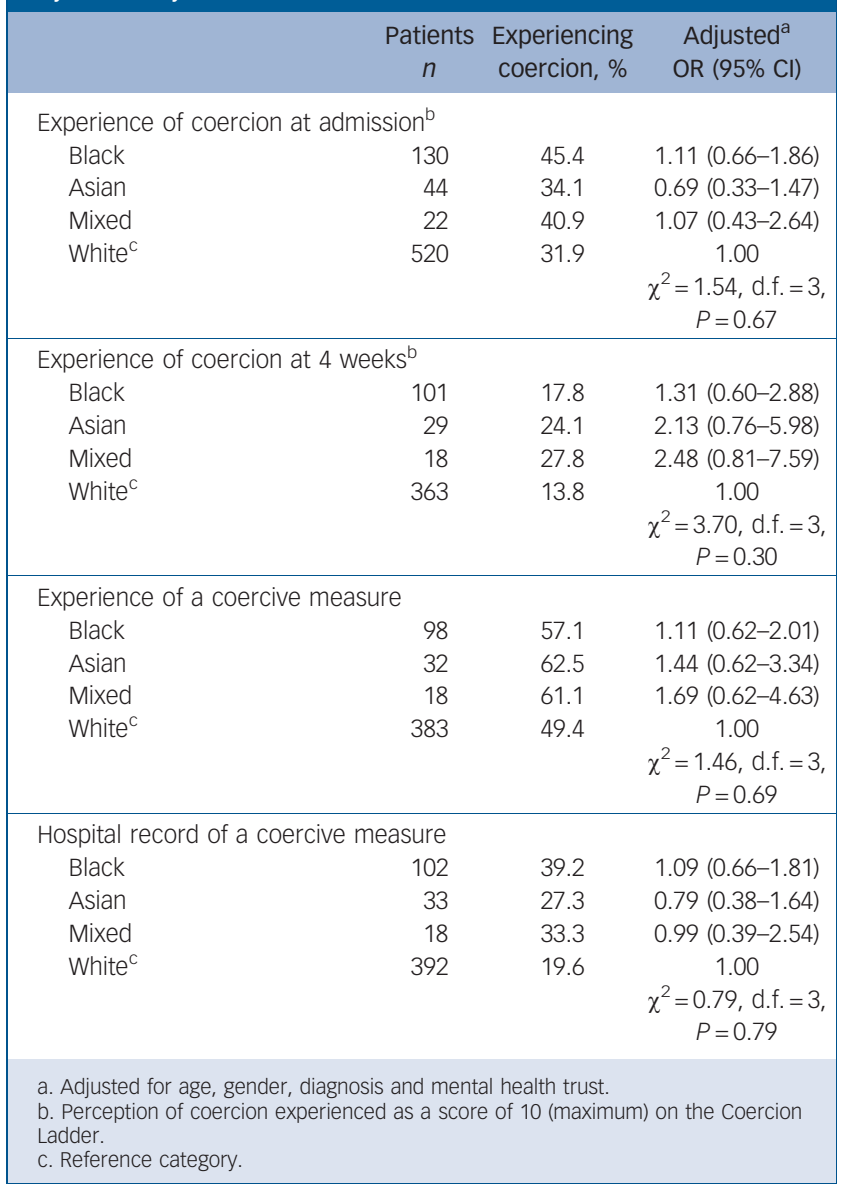

\section{Discussion}

We found no association between ethnicity and patient perception of coercion at admission or during the first 4 weeks after admission. There was also no association between ethnicity and either self-report or hospital record of a coercive measure being used during the first 4 weeks of hospital stay. The treating mental health trust was strongly associated both with patient experience of coercion at admission and with whether a coercive measure was recorded in the patient's notes. People from ethnic minorities, particularly Black patients, were more likely to be in hospitals that were perceived to be more coercive. This explained the apparent association between BME and coercion in some unadjusted analyses and highlights the importance of adjusting for factors that may explain apparent associations. Our results on coercive practices do not support the suggestion that institutional racism is common in UK mental health services. However, the wide confidence intervals mean that we cannot exclude the possibility of a clinically important association between some of these coercive factors and ethnicity, and the generalisability of these results also needs to be investigated.

A strength of this study was our sample size and the number and geographical spread of mental health trusts involved in patient recruitment. Previous studies of ethnicity and coercion in the UK recruited only from hospital wards in London and relied on routine hospital records; or, where both patient self-report and information from notes were collected, the focus was on the admission process and the sample size was small. ${ }^{4,5,10}$ Sample size was also small in the US study. ${ }^{6}$ Although we recruited only half of the eligible population, response rates across ethnic groups were similar and this is reassuring in relation to the validity of our results.

Ethnic groupings are always artificial, but are potentially useful in identifying the influence of racist stereotypes on mental health practice. ${ }^{11}$ Our results suggest that improvements are needed in mental health trusts where there are higher rates of involuntary admission of BME patients. Although such differences across trusts may relate to policy or staff training, they are likely to reflect the characteristics of the catchment area. Our results suggest that patients from areas with a high proportion of ethnic minorities are more likely to experience coercion, regardless of whether they themselves belong to an ethnic minority.

\section{Olive Bennewith, BA, Tim Amos, MRCPsych, Glyn Lewis, FRCPsych, Academic Unit of Psychiatry, University of Bristol; Christina Katsakou, PhD, Unit for Social and Community Psychiatry, Barts and The London School of Medicine, Queen Mary, University of London; Til Wykes, PhD, Department of Psychology, Institute of Psychiatry, London; Richard Morriss, MD, University of Nottingham, Division of Psychiatry, Nottingham; Stefan Priebe, FRCPsych, Unit for Social and Community Psychiatry, Barts and The London School of Medicine, Queen Mary, University of London, UK \\ Correspondence: Olive Bennewith, Academic Unit of Psychiatry, Cotham House, Cotham Hill, Bristol BS6 6JL, UK. Email: O.M.Bennewith@bristol.ac.uk \\ First received 26 Mar 2009, final revision 28 Aug 2009, accepted 26 Sep 2009}

\section{Funding}

The study was funded by a grant from the Policy Research Programme of the UK Department of Health, Commission No. 0230072, and supported by the National Institute for Health Research UK Mental Health Research Network. The project was coordinated by the Unit for Social and Community Psychiatry, Barts and The London School of Medicine and Dentistry, Queen Mary University of London, which received funding from the Department of Health. The views expressed here are those of the authors and not necessarily those of the Department of Health.

\section{Acknowledgements}

We are grateful to all interviewed patients and staff of the participating trusts. We also thank Louisa Bolt, Simon Clarke, Susan Collinson, Suzi Curtis, Rosemary Davies, Rebekka Evans, Sarah Grant, Dannielle Oliver, Felicitas Rost, Rebecca Read, Rajinder Sidhu, Emma Williams and Jessica Zetteler for their contribution to the study.

\section{References}

1 Norfolk, Suffolk, Cambridgeshire Health Authority. Independent Inquiry into the Death of David Bennett. Cambridgeshire Health Authority, 2003.

2 Sabshin M, Diesenhaus $H$, Wilkeson R. Dimensions of institutional racism in psychiatry. Am J Psychiatry 1970; 127: 787-93.

3 Sashidharan SP. Institutional racism in British psychiatry. Psychiatr Bull 2001; 25: 244-7.

4 Gudjonsson GH, Rabe-Hesketh S, Szmukler G. Management of psychiatric in-patient violence: patient ethnicity and use of medication, restraint and seclusion. Br J Psychiatry 2004; 184: 258-62.

5 Bowers L, Douzenis A, Galeazzi GM, Forghieri M, Tsopelas C, Simpson A, et al. Disruptive and dangerous behaviour on acute psychiatric wards in three European centres. Soc Psychiatry Psychiatr Epidemiol 2005; 40: 822-8.

6 Flaherty J, Meager R. Measuring racial bias in inpatient treatment. Am J Psychiatry 1980; 137: 679-82.

7 Priebe S, Katsakou C, Amos T, Leese M, Morriss R, Rose D, et al. Patients views and readmissions 1 year after involuntary hospitalisation. $\mathrm{Br} J$ Psychiatry 2009; 194: 49-54.

8 Iversen KI, Høyer G, Sexton H, Grønli OK. Perceived coercion among patients admitted to acute wards in Norway. Nord J Psychiatry 2002; 56: 433-9.

9 Cantril H. The Pattern of Human Concerns. Rutgers University Press, 1965.

10 Bindman J, Reid Y, Szmukler G, Tiller J, Thornicroft G, Leese M. Perceived coercion at admission to psychiatric hospital and engagement with followup: a cohort study. Soc Psychiatry Psychiatr Epidemiol 2005; 40: 160-6.

11 Singh SP. Ethnicity in psychiatric epidemiology: need for precision. $\mathrm{Br} J$ Psychiatry 1997; 171: 305-8. 